\title{
Recurrent form of the renormalization group relations for the higher-order hadronic vacuum polarization function perturbative expansion coefficients
}

\author{
A.V. Nesterenko \\ Bogoliubov Laboratory of Theoretical Physics, Joint Institute for Nuclear Research, \\ Dubna, 141980, Russian Federation
}

\begin{abstract}
The renormalization group relations for the higher-order hadronic vacuum polarization function perturbative expansion coefficients are studied. The folded recurrent and unfolded explicit forms of such relations are obtained. The explicit expression for the coefficients, which incorporate the contributions of the $\pi^{2}$-terms in the perturbative expansion of the $R$-ratio of electron-positron annihilation into hadrons, is derived at an arbitrary loop level. The obtained results can be employed as an independent crosscheck of the higher-order perturbative calculations of the hadronic vacuum polarization function and in the studies of the renormalization scale setting in the relevant physical observables.
\end{abstract}

Keywords: renormalization group, hadronic vacuum polarization function

\section{Introduction}

A variety of issues of elementary particle physics is inherently based on the hadronic vacuum polarization function $\Pi\left(q^{2}\right)$, the corresponding Adler function $D\left(Q^{2}\right)$, and the function $R(s)$. In particular, these functions play a key role in the theoretical studies of numerous strong interaction processes, for example, electron-positron annihilation into hadrons, inclusive $\tau$ lepton hadronic decay, and hadronic contributions to such precise electroweak observables as the muon anomalous magnetic moment and the running of the electromagnetic coupling. Basically, all these processes are of a straight relevance to the future collider projects [1-7], contemporary experiments [8 12], and various ongoing research programs, see, e.g., Refs. [13, 14]. The theoretical investigation of these issues provides a decisive consistency test of the Standard Model and imposes firm restrictions on a possible new physics beyond the latter. In turn, an anticipated increase of the accuracy of measurements at the future experimental facilities calls for a further refinement of theoretical approaches, which address both perturbative and intrinsically nonperturbative aspects of hadron dynamics.

Factually, the perturbation theory and the renormalization group (RG) method remain to be a basic tool for the theoretical exploration of Quantum Chromodynamics (QCD) at high energies. Specifically, the calculation of pertinent Feynman diagrams constitutes a reliable 
way to obtain the perturbative expression for the hadronic vacuum polarization function of the form of Eq. (8) below. At the same time, the RG method also enables one to tie together the higher-order coefficients $\Pi_{j, k}$ entering Eq. (8) . At the few lowest orders of perturbation theory these RG relations assume a rather simple form, whereas at the higher orders such relations become quite cumbersome and their derivation requires considerable efforts.

The primary objective of the paper is to obtain the RG relations for the higher-order hadronic vacuum polarization function perturbative expansion coefficients $\Pi_{j, k}$ in a folded recurrent and unfolded explicit forms. It is also of an apparent interest to derive, at an arbitrary loop level, the explicit expression for the coefficients embodying the $\pi^{2}$-terms in the perturbative expansion of the $R$-ratio of electron-positron annihilation into hadrons, which play a substantial role for the subject on hand.

The layout of the paper is as follows. Section 2 delineates the hadronic vacuum polarization function $\Pi\left(q^{2}\right)$, the Adler function $D\left(Q^{2}\right)$, and the function $R(s)$, recaps their perturbative expressions, and expounds the corresponding RG equations. In Sect. 3 the RG relations for the higher-order hadronic vacuum polarization function perturbative expansion coefficients $\Pi_{j, k}$ are obtained in a folded recurrent and unfolded explicit forms, the explicit expression for the coefficients, which incorporate the $\pi^{2}$-terms in the perturbative expansion of the $R$-ratio of electron-positron annihilation into hadrons, is derived at an arbitrary loop level, and the discussion of practical applications of the obtained results is presented. Section 4 summarizes the basic results. Appendix $\mathrm{A}$ contains a supplementary material.

\section{Methods}

\subsection{Functions $\Pi\left(q^{2}\right), D\left(Q^{2}\right)$, and $R(s)$}

As discussed earlier, the theoretical study of a broad pattern of the strong interaction processes is inherently based on the hadronic vacuum polarization function $\Pi\left(q^{2}\right)$, which is defined as the scalar part of the hadronic vacuum polarization tensor

$$
\Pi_{\mu \nu}\left(q^{2}\right)=i \int d^{4} x e^{i q x}\left\langle 0\left|T\left\{J_{\mu}(x) J_{\nu}(0)\right\}\right| 0\right\rangle=\frac{i}{12 \pi^{2}}\left(q_{\mu} q_{\nu}-g_{\mu \nu} q^{2}\right) \Pi\left(q^{2}\right),
$$

with $q^{2}<0$ being the spacelike kinematic variable. The function $\Pi\left(q^{2}\right)$ (1) satisfies the inhomogeneous RG equation

$$
\left[\frac{\partial}{\partial \ln \mu^{2}}+\frac{\partial a_{\mathrm{s}}\left(\mu^{2}\right)}{\partial \ln \mu^{2}} \frac{\partial}{\partial a_{\mathrm{s}}}\right] \Pi\left(q^{2}, \mu^{2}, a_{\mathrm{s}}\right)=\gamma\left(a_{\mathrm{s}}\right)
$$

where $\mu^{2}>0$ denotes the renormalization scale and $\gamma\left(a_{\mathrm{s}}\right)$ stands for the anomalous dimension. In Eq. (2) $a_{\mathrm{s}}\left(\mu^{2}\right)=\alpha_{\mathrm{s}}\left(\mu^{2}\right) \beta_{0} /(4 \pi)$ is the so-called QCD couplant, which satisfies the RG equation

$$
\frac{\partial a_{\mathrm{s}}\left(\mu^{2}\right)}{\partial \ln \mu^{2}}=\beta\left(a_{\mathrm{s}}\right)
$$

where $\beta\left(a_{\mathrm{s}}\right)$ denotes the $\beta$ function, $\beta_{0}=11-2 n_{f} / 3$ is the one-loop $\beta$ function perturbative expansion coefficient, and $n_{f}$ stands for the number of active flavors. For practical applica- 
tions it is particularly convenient to deal with the Adler function, which is defined as the logarithmic derivative of the hadronic vacuum polarization function [15]

$$
D\left(Q^{2}\right)=-\frac{d \Pi\left(-Q^{2}\right)}{d \ln Q^{2}}
$$

with $Q^{2}=-q^{2}>0$ being the spacelike kinematic variable. The Adler function satisfies the homogeneous RG equation

$$
\left[\frac{\partial}{\partial \ln \mu^{2}}+\frac{\partial a_{\mathrm{s}}\left(\mu^{2}\right)}{\partial \ln \mu^{2}} \frac{\partial}{\partial a_{\mathrm{s}}}\right] D\left(Q^{2}, \mu^{2}, a_{\mathrm{s}}\right)=0
$$

In turn, the function $R(s)$, which is commonly identified with the $R$-ratio of electronpositron annihilation into hadrons $R(s)=\sigma\left(e^{+} e^{-} \rightarrow\right.$ hadrons; $\left.s\right) / \sigma\left(e^{+} e^{-} \rightarrow \mu^{+} \mu^{-} ; s\right)$, can be calculated as the discontinuity of the hadronic vacuum polarization function across the physical cut

$$
R(s)=\frac{1}{2 \pi i} \lim _{\varepsilon \rightarrow 0_{+}}[\Pi(s+i \varepsilon)-\Pi(s-i \varepsilon)]=\frac{1}{\pi} \operatorname{Im}_{\varepsilon \rightarrow 0_{+}} \lim _{\varepsilon}(s+i \varepsilon)
$$

or, equivalently, by integrating Eq. (44) in finite limits, specifically [16, 17]

$$
R(s)=\frac{1}{2 \pi i} \lim _{\varepsilon \rightarrow 0_{+}} \int_{s+i \varepsilon}^{s-i \varepsilon} D(-\zeta) \frac{d \zeta}{\zeta}
$$

where $s=q^{2}>0$ denotes the timelike kinematic variable, namely, the center-of-mass energy squared. In Eq. (77) the integration contour in the complex $\zeta$-plane lies in the region of analyticity of the integrand. The complete set of relations, which express the functions on hand in terms of each other, as well as a detailed discussion of their physical implications can be found in, e.g., Ref. [18] and references therein.

\section{$2.2 \quad$ Functions $\Pi\left(q^{2}\right), D\left(Q^{2}\right)$, and $R(s)$ within perturbation theory}

In the framework of perturbation theory the $\ell$-loop expression for the hadronic vacuum polarization function (11) can be represented as

$$
\Pi^{(\ell)}\left(q^{2}, \mu^{2}, a_{\mathrm{s}}\right)=\sum_{j=0}^{\ell}\left[a_{\mathrm{s}}^{(\ell)}\left(\mu^{2}\right)\right]^{j} \sum_{k=0}^{j+1} \Pi_{j, k} \ln ^{k}\left(\frac{\mu^{2}}{-q^{2}}\right), \quad q^{2} \rightarrow-\infty
$$

and, as immediatedly follows from Eq. (44), the corresponding expression for the Adler function reads

$$
D^{(\ell)}\left(Q^{2}, \mu^{2}, a_{\mathrm{s}}\right)=\sum_{j=0}^{\ell}\left[a_{\mathrm{s}}^{(\ell)}\left(\mu^{2}\right)\right]^{j} \sum_{k=0}^{j+1} k \Pi_{j, k} \ln ^{k-1}\left(\frac{\mu^{2}}{Q^{2}}\right), \quad Q^{2} \rightarrow \infty .
$$

Note that the common prefactor $N_{\mathrm{c}} \sum_{f=1}^{n_{f}} Q_{f}^{2}$ is omitted throughout, where $N_{\mathrm{c}}=3$ denotes the number of colors and $Q_{f}$ stands for the electric charge of $f$-th quark. The native choice 
of the renormalization scale $\mu^{2}=Q^{2}$ (that amounts to the RG summation in the spacelike domain) casts Eq. (9) to a well-known form $\left(\Pi_{0,1}=1\right)$

$$
D^{(\ell)}\left(Q^{2}\right)=\sum_{j=0}^{\ell} \Pi_{j, 1}\left[a_{\mathrm{s}}^{(\ell)}\left(Q^{2}\right)\right]^{j}=1+\sum_{j=1}^{\ell} d_{j}\left[a_{\mathrm{s}}^{(\ell)}\left(Q^{2}\right)\right]^{j}, \quad d_{j}=\Pi_{j, 1},
$$

whereas the elimination of dependence of Eq. (8) on the renormalization scale was discussed in, e.g., Refs. [18 23]. It is worthwhile to mention here that a general choice of the renormalization scale $\mu^{2}=c Q^{2}$ (with $c \neq 1$ being a positive constant) keeps in the resulting expression for the Adler function $D^{(\ell)}\left(Q^{2}\right)$ all the terms proportional to the higher-order coefficients $\Pi_{j, k}(0 \leq j \leq \ell, 0 \leq k \leq j+1)$ appearing on the right-hand side of Eq. (9). In turn, at the $\ell$-loop level the anomalous dimension and $\beta$ function entering Eqs. (2) and (5) take the following form

$$
\begin{gathered}
\gamma^{(\ell)}\left(a_{\mathrm{s}}\right)=\sum_{j=0}^{\ell} \gamma_{j}\left[a_{\mathrm{s}}^{(\ell)}\left(\mu^{2}\right)\right]^{j}, \\
\beta^{(\ell)}\left(a_{\mathrm{s}}\right)=-\sum_{i=0}^{\ell-1} B_{i}\left[a_{\mathrm{s}}^{(\ell)}\left(\mu^{2}\right)\right]^{i+2}, \quad B_{i}=\frac{\beta_{i}}{\beta_{0}^{i+1}} .
\end{gathered}
$$

The explicit expressions for the perturbative expansion coefficients $\Pi_{j, k}$ (8) and $\gamma_{j}$ (11) are currently available up to the fourth order in the QCD couplant $a_{\mathrm{s}}$, whereas the coefficients $\beta_{i}$ (12) are known up to the fifth order in $a_{\text {s }}$, see Refs. [24, 25] and [26], respectively.

As for the function $R(s)$, it can be calculated by making use of either of Eqs. (6) and (7). At the same time, it is necessary to emphasize here that the RG summation must be performed in Eqs. (8) and (9) in the spacelike domain prior to the application of Eqs. (6) and (7), respectively. Otherwise the effects due to continuation of theoretical results from spacelike into timelike domain may not be properly accounted for. In particular, the direct calculation of discontinuity (6) of expression (8) with subsequent assignment of the renormalization scale $\mu^{2}=|s|$ (that factually amounts to an incomplete RG summation in the timelike domain, see, e.g., Refs. [20,21, 23, 27]) yields

$$
R^{(\ell)}(s)=1+\sum_{j=1}^{\ell} r_{j}\left[a_{\mathrm{s}}^{(\ell)}(|s|)\right]^{j}, \quad r_{j}=d_{j}-\delta_{j}
$$

where $d_{j}=\Pi_{j, 1}$ stand for the Adler function perturbative expansion coefficients (10) and $\delta_{j}$ embody the contributions of the so-called $\pi^{2}$-terms (which play a significant role in the studies of the process on hand), namely

$$
\delta_{1}=0, \quad \delta_{2}=0, \quad \delta_{j}=\sum_{k=1}^{K(j)}(-1)^{k+1} \pi^{2 k} \Pi_{j, 2 k+1}, \quad j \geq 3 .
$$

In this equation

$$
K(n)=\frac{n-2}{2}+\frac{n \bmod 2}{2}
$$

and $(n \bmod m)$ is the remainder on division of $n$ by $m$. It is worthwhile to note also that the function $R^{(\ell)}(s)(13)$ only partially retains the effects due to continuation of theoretical results from spacelike into timelike domain and has certain shortcomings, see, e.g., Refs. [1618, 20, 21, 23, 27, 29] and references therein for a detailed discussion of these issues. 


\section{Results and discussion}

\subsection{Recurrence relations for the coefficients $\Pi_{j, k}$}

As mentioned earlier, the RG equations for the functions on hand bind together the higherorder perturbative coefficients $\Pi_{j, k}$ appearing in Eqs. (8) and (9). In particular, at an arbitrary loop level the coefficients $\Pi_{j, k}(j \geq 1 ; k=1, \ldots, j+1)$ can be expressed in terms of the coefficients $\gamma_{i}(i=1, \ldots, j)$ entering Eq. (11) and (if $\left.j \geq 2\right) \Pi_{i, 0}(i=1, \ldots, j-1$ ). Specifically, Eqs. (22), (3), (8), (11), and (12) imply that

$$
\begin{aligned}
& \sum_{j=0}^{\ell}\left[a_{\mathrm{s}}^{(\ell)}\left(\mu^{2}\right)\right]^{j}\left\{\left[\sum_{k=0}^{j+1} k \Pi_{j, k} \ln ^{k-1}\left(\frac{\mu^{2}}{-q^{2}}\right)\right]-\gamma_{j}\right\}= \\
& =\left\{\sum_{j=0}^{\ell} j\left[a_{\mathrm{s}}^{(\ell)}\left(\mu^{2}\right)\right]^{j-1} \sum_{k=0}^{j+1} \Pi_{j, k} \ln ^{k}\left(\frac{\mu^{2}}{-q^{2}}\right)\right\}\left\{\sum_{i=0}^{\ell-1} B_{i}\left[a_{\mathrm{s}}^{(\ell)}\left(\mu^{2}\right)\right]^{i+2}\right\} .
\end{aligned}
$$

For example, at the first few orders of perturbation theory this equation yields $\Pi_{0,1}=\gamma_{0}$, $\Pi_{1,1}=\gamma_{1}, \Pi_{2,1}=\Pi_{1,0}+\gamma_{2}, \Pi_{2,2}=\gamma_{1} / 2\left(\Pi_{j, j+1}=0\right.$ for $\left.j \geq 1\right)$, see, e.g., Refs. [23, 25], whereas at the higher-loop levels the corresponding RG relations for the coefficients $\Pi_{j, k}$ become quite tangled and can be found in Appendix A of Ref. [23].

At the same time, the RG equation for the Adler function (5) provides the relations for the higher-order perturbative expansion coefficients $\Pi_{j, k}$, which, being equivalent to the aforementioned ones, have a somewhat different form. Namely, at any given loop level Eqs. (5) , (3), (9), and (12) enable one to express the coefficients $\Pi_{j, k}(j \geq 2 ; k=2, \ldots, j+1)$ in terms of the coefficients $\Pi_{i, 1}(i=1, \ldots, j-1)$, specifically

$$
\begin{aligned}
& \sum_{j=0}^{\ell}\left[a_{\mathrm{s}}^{(\ell)}\left(\mu^{2}\right)\right]^{j}\left[\sum_{k=0}^{j+1} k(k-1) \Pi_{j, k} \ln ^{k-2}\left(\frac{\mu^{2}}{Q^{2}}\right)\right]= \\
& =\left\{\sum_{j=0}^{\ell} j\left[a_{\mathrm{s}}^{(\ell)}\left(\mu^{2}\right)\right]^{j-1} \sum_{k=0}^{j+1} k \Pi_{j, k} \ln ^{k-1}\left(\frac{\mu^{2}}{Q^{2}}\right)\right\}\left\{\sum_{i=0}^{\ell-1} B_{i}\left[a_{\mathrm{s}}^{(\ell)}\left(\mu^{2}\right)\right]^{i+2}\right\} .
\end{aligned}
$$

In particular, at the first few orders of perturbation theory Eq. (17) yields $\Pi_{2,2}=\Pi_{1,1} / 2$, $\Pi_{3,2}=\Pi_{1,1}\left(B_{1} / 2\right)+\Pi_{2,1}, \Pi_{3,3}=\Pi_{1,1} / 3\left(\Pi_{j, j+1}=0\right.$ for $\left.j \geq 1\right)$, whereas the RG relations for the coefficients $\Pi_{j, k}$ at the higher orders become rather cumbrous and are given in Appendix A.

At an arbitrary loop level the RG relations for the coefficients $\Pi_{j, k}$, which follow from Eq. (17), can be represented in a compact recurrent form, namely

$$
\begin{gathered}
\Pi_{j, 2}=\frac{1}{2} \sum_{i=1}^{j-1} i B_{j-i-1} \Pi_{i, 1}, \quad j \geq 2, \\
\Pi_{j, k}=\frac{1}{T_{k-1}} \sum_{i=k-2}^{j-2} i(i+j) \mathfrak{B}_{j-i-2} \Pi_{i, k-2}, \quad j \geq k, \quad k \geq 3,
\end{gathered}
$$


where

$$
\mathfrak{B}_{n}=\frac{1}{4} \sum_{i=0}^{n} B_{i} B_{n-i}, \quad B_{i}=\frac{\beta_{i}}{\beta_{0}^{i+1}}
$$

and

$$
T_{n}=\frac{1}{2} n(n+1)
$$

denotes the $\mathrm{so}^{-}$-called triangular number.

The obtained recurrence relations (18) and (19) can also be unfolded, that eventually results in the following explicit expressions for the higher-order coefficients $\Pi_{j, k}$ for, respectively, odd and even values of the second index:

$$
\begin{aligned}
& \Pi_{j, 2 k+1}= \frac{2^{k}}{(2 k+1) !} \sum_{i_{1}=2(k-1)+1}^{\sum_{i_{2}=2(k-2)+1}^{j-2} \ldots \sum_{i_{n}=2(k-n)+1}^{i_{1}-2} \cdots \sum_{i_{k}=1}^{i_{n-1}-2}}\left(j+i_{1}\right) i_{1} \times \\
& \times \underbrace{i_{k-1}-2}_{(k-1) \text { sums }}(k-1) \text { products } \\
& \times \mathfrak{B}_{j-i_{1}-2}^{\left(i_{1}+i_{2}\right) i_{2} \times \ldots \times\left(i_{n-1}+i_{n}\right) i_{n} \times \ldots \times\left(i_{k-1}+i_{k}\right) i_{k}} \times \\
& \underbrace{\mathfrak{B}_{i_{1}-i_{2}-2} \ldots \mathfrak{B}_{i_{n-1}-i_{n}-2} \ldots \mathfrak{B}_{i_{k-1}-i_{k}-2}}_{(k-1) \text { terms }} \prod_{i_{k}, 1}, \quad j \geq(2 k+1), \quad k \geq 1,
\end{aligned}
$$

$$
\begin{aligned}
\Pi_{j, 2 k}= & \frac{2^{k-1}}{(2 k) !} \sum_{i_{1}=2(k-1)}^{j-2} \underbrace{\sum_{i_{2}=2(k-2)}^{i_{1}-2} \ldots \sum_{i_{n}=2(k-n)}^{i_{n-1}-2} \ldots \sum_{i_{k-1}=2}^{i_{k-2}-2} \sum_{i_{k}=1}^{i_{k-1}-1}\left(j+i_{1}\right) i_{1} \times}_{(k-2) \text { sums }} \\
& \times \underbrace{\left(i_{1}+i_{2}\right) i_{2} \times \ldots \times\left(i_{n-1}+i_{n}\right) i_{n} \times \ldots \times\left(i_{k-2}+i_{k-1}\right) i_{k-1}}_{(k-2) \text { products }} \times i_{k} \times \\
& \times \mathfrak{B}_{j-i_{1}-2} \underbrace{\mathfrak{B}_{i_{1}-i_{2}-2} \ldots \mathfrak{B}_{i_{n-1}-i_{n}-2} \ldots \mathfrak{B}_{i_{k-2}-i_{k-1}-2}}_{(k-2) \text { terms }} B_{i_{k-1}-i_{k}-1} \Pi_{i_{k}, 1}, \quad j \geq 2 k, \quad k \geq 2,
\end{aligned}
$$

where the coefficients $\mathfrak{B}_{n}$ and $B_{i}$ are defined in Eq. (20). It is straightforward to verify that Eqs. (22) and (23) reproduce the RG relations for the coefficients $\Pi_{j, k}$, which follow from Eq. (17), see Appendix A. It is worthwhile to mention that in certain cases Eqs. (18), (22), and (23) can be represented in a somewhat simpler form, in particular,

$$
\Pi_{j, j-1}=\left(H_{j-1}-1\right) B_{1} \Pi_{1,1}+\Pi_{2,1}, \quad j \geq 3 ; \quad \Pi_{j, j}=\frac{1}{j} \Pi_{1,1}, \quad j \geq 2,
$$

where

$$
H_{n}=\sum_{k=1}^{n} \frac{1}{k}
$$

stands for the so-called harmonic number. 
At the same time, the higher-order coefficients $\Pi_{j, k}$ can also be expressed in terms of the coefficients $\Pi_{i, 0}$ and $\gamma_{i}$ (11). Specifically, for this purpose the obtained results should be supplemented by the relations [23]

$$
\Pi_{0,1}=\gamma_{0}, \quad \Pi_{1,1}=\gamma_{1}, \quad \Pi_{j, 1}=\gamma_{j}+\sum_{k=1}^{j-1} k \Pi_{k, 0} B_{j-k-1}, \quad B_{j}=\frac{\beta_{j}}{\beta_{0}^{j+1}}, \quad j \geq 2 .
$$

In particular, Eqs. (18), (22), (23), and (26), being applied jointly, reproduce the $\mathrm{RG}$ relations for the coefficients $\Pi_{j, k}$, which follow from Eq. (16), see Appendix A of Ref. [23].

\subsection{Discussion}

First of all, it is necessary to outline that the results obtained in Sect. 3.1 can be employed as an independent crosscheck for the perturbative calculations at the higher loop levels. In particular, Eqs. (18), (22) , (23) enable one to find explicit expressions for all the coefficients $\Pi_{j, k}$, which involve the hitherto known perturbative coefficients $\Pi_{i, 1}$. As mentioned earlier, the latter are currently available up to the fourth order in the strong running coupling $(i=0, \ldots, 4)$, therefore Eqs. (18), (22), (23) provide, at an arbitrary loop level (i.e., for any given $j \geq 2$ ), the explicit expressions for the higher-order coefficients $\Pi_{j, j-n}(n=0, \ldots, \min \{\mathrm{j}-2,3\})$. For example, at the 10th and 25th orders of perturbation theory

$$
\begin{gathered}
\Pi_{10,9}=\frac{4609}{2520} B_{1} \Pi_{1,1}+\Pi_{2,1}, \quad \Pi_{10,10}=\frac{1}{10} \Pi_{1,1}, \\
\Pi_{25,24}=\frac{990874363}{356948592} B_{1} \Pi_{1,1}+\Pi_{2,1}, \quad \Pi_{25,25}=\frac{1}{25} \Pi_{1,1},
\end{gathered}
$$

where (see Refs. [19, 30,32])

$$
\begin{gathered}
\Pi_{1,1}=\frac{4}{\beta_{0}}, \quad \Pi_{2,1}=\left(\frac{4}{\beta_{0}}\right)^{2}\left\{\frac{365}{24}-11 \zeta(3)+n_{f}\left[-\frac{11}{12}+\frac{2}{3} \zeta(3)\right]\right\}, \\
B_{j}=\frac{\beta_{j}}{\beta_{0}^{j+1}}, \quad \beta_{0}=11-\frac{2}{3} n_{f}, \quad \beta_{1}=102-\frac{38}{3} n_{f},
\end{gathered}
$$

and $\zeta(3) \simeq 1.2021$ stands for the Riemann $\zeta$ function.

Additionally, Eq. (22) enables one to obtain, at an arbitrary loop level, the explicit expression for the coefficients $\delta_{j}$ (14) embodying the contributions of the $\pi^{2}$-terms in the perturbative expression for the $R$-ratio of electron-positron annihilation into hadrons (13), namely $\left(\delta_{1}=\delta_{2}=0\right)$

$$
\begin{aligned}
\delta_{j}= & -\sum_{k=1}^{K(j)} \frac{\left(-2 \pi^{2}\right)^{k}}{(2 k+1) !} \sum_{i_{1}=2(k-1)+1}^{j-2} \underbrace{\sum_{i_{2}=2(k-2)+1}^{i_{1}-2} \ldots \sum_{i_{n}=2(k-n)+1}^{i_{n-1}-2} \cdots \sum_{i_{k}=1}^{i_{k-1}-2}}_{(k-1) \mathrm{sums}}\left(j+i_{1}\right) i_{1} \times \\
& \times \underbrace{\left(i_{1}+i_{2}\right) i_{2} \times \ldots \times\left(i_{n-1}+i_{n}\right) i_{n} \times \ldots \times\left(i_{k-1}+i_{k}\right) i_{k}}_{(k-1) \text { products }} \times \\
& \times \mathfrak{B}_{j-i_{1}-2} \underbrace{\mathfrak{B}_{i_{1}-i_{2}-2} \ldots \text { terms }}_{\mathfrak{B}_{i_{n-1}-i_{n}-2} \ldots \mathfrak{B}_{i_{k-1}-i_{k}-2}} d_{i_{k}}, \quad d_{j}=\Pi_{j, 1}, \quad j \geq 3,
\end{aligned}
$$


where the function $K(n)$ is defined in Eq. (15) and the coefficients $\mathfrak{B}_{n}$ are specified in Eq. (20), see also Refs. [18, 23, 27] and references therein for a detailed discussion of this issue. It is straightforward to verify that Eq. (31) reproduces all the explicit expressions for the coefficients $\delta_{j}$ obtained earlier by making use of other methods, see, in particular, Appendix $\mathrm{C}$ of Ref. [18], Sect. 4 of Ref. [27], and Sect. 2.3 of Ref. [23].

As mentioned above, the $\pi^{2}$-terms play a valuable role in the studies of electron-positron annihilation into hadrons and the related physical observables. Specifically, as demonstrated in Ref. [23], the higher-order $\pi^{2}$-terms (31) make a significant effect on the evaluated strong running coupling and the QCD scale parameter in the energy range of current BESIII experiment [11]. In turn, the effect of the higher-order $\pi^{2}$-terms (31) on the resulting value of the strong running coupling in the future ILC experiment energy range [3] and the corresponding effect on the $R$-ratio itself in the future CLIC experiment energy range [4] are studied in Refs. [23] and [27], respectively.

At the same time, as discussed in Sect. 2.2, for a general choice of the renormalization scale $\mu^{2}$ all the higher-order coefficients $\Pi_{j, k}$ contribute to the Adler function. Thus, the obtained results (18)-(23) can also be employed in the studies of the renormalization scale setting in the theoretical expressions for the relevant physical observables, such as, e.g., the $R$-ratio of electron-positron annihilation into hadrons in the energy range of current BESIII [11] and KEDR [12] experiments.

\section{Conclusions}

The renormalization group relations for the higher-order hadronic vacuum polarization function perturbative expansion coefficients $\Pi_{j, k}$ are studied. The folded recurrent [Eqs. (18), (19)] and unfolded explicit [Eqs. (22), (23)] forms of such relations are obtained. The explicit expression for the coefficients $\delta_{j}$ [Eq. (31)] embodying the contributions of the $\pi^{2}$-terms in the perturbative expansion of the $R$-ratio of electron-positron annihilation into hadrons, which play a substantial role in the studies of the process on hand, is derived at an arbitrary loop level. The obtained results can also be employed as an independent crosscheck of the higher-order perturbative calculations of the hadronic vacuum polarization function and in the studies of the renormalization scale setting in the relevant physical observables.

\section{A RG relations for the coefficients $\Pi_{j, k}$}

As discussed in Sect. 3.1, at any given order $j$ the hadronic vacuum polarization function perturbative expansion coefficients $\Pi_{j, k}(j \geq 2 ; k=2, \ldots, j+1)$ entering Eq. (9) can be expressed in terms of the coefficients $\Pi_{i, 1}(i=1, \ldots, j-1)$ by making use of the RG equation (17). The corresponding relations for the coefficients $\Pi_{j, k}$ at the first eight loop levels are presented in the following $\left(\Pi_{j, j+1}=0\right.$ for $\left.j \geq 1\right)$.

$$
\Pi_{j, 2}=\frac{1}{2} \sum_{i=1}^{j-1} i B_{j-i-1} \Pi_{i, 1}, \quad \Pi_{j, j}=\frac{1}{j} \Pi_{1,1}, \quad j \geq 2 .
$$




$$
\begin{aligned}
& \Pi_{4,3}=\frac{5}{6} B_{1} \Pi_{1,1}+\Pi_{2,1} . \\
& \Pi_{5,3}=\left(\frac{1}{2} B_{1}^{2}+B_{2}\right) \Pi_{1,1}+\frac{7}{3} B_{1} \Pi_{2,1}+2 \Pi_{3,1} \\
& \Pi_{5,4}=\frac{13}{12} B_{1} \Pi_{1,1}+\Pi_{2,1} . \\
& \Pi_{6,3}=\frac{7}{6}\left(B_{1} B_{2}+B_{3}\right) \Pi_{1,1}+\frac{8}{3}\left(\frac{1}{2} B_{1}^{2}+B_{2}\right) \Pi_{2,1}+\frac{9}{2} B_{1} \Pi_{3,1}+\frac{10}{3} \Pi_{4,1}, \\
& \Pi_{6,4}=\left(\frac{35}{24} B_{1}^{2}+\frac{3}{2} B_{2}\right) \Pi_{1,1}+\frac{47}{12} B_{1} \Pi_{2,1}+\frac{5}{2} \Pi_{3,1}, \\
& \Pi_{6,5}=\frac{77}{60} B_{1} \Pi_{1,1}+\Pi_{2,1} . \\
& \Pi_{7,3}=\frac{4}{3}\left(B_{1} B_{3}+\frac{1}{2} B_{2}^{2}+B_{4}\right) \Pi_{1,1}+3\left(B_{1} B_{2}+B_{3}\right) \Pi_{2,1}+ \\
& +5\left(\frac{1}{2} B_{1}^{2}+B_{2}\right) \Pi_{3,1}+\frac{22}{3} B_{1} \Pi_{4,1}+5 \Pi_{5,1}, \\
& \Pi_{7,4}=\left(\frac{5}{8} B_{1}^{3}+\frac{23}{6} B_{1} B_{2}+2 B_{3}\right) \Pi_{1,1}+\left(\frac{59}{12} B_{1}^{2}+5 B_{2}\right) \Pi_{2,1}+\frac{37}{4} B_{1} \Pi_{3,1}+5 \Pi_{4,1}, \\
& \Pi_{7,5}=\left(\frac{17}{6} B_{1}^{2}+2 B_{2}\right) \Pi_{1,1}+\frac{57}{10} B_{1} \Pi_{2,1}+3 \Pi_{3,1} \\
& \Pi_{7,6}=\frac{29}{20} B_{1} \Pi_{1,1}+\Pi_{2,1} . \\
& \Pi_{8,3}=\frac{3}{2}\left(B_{1} B_{4}+B_{2} B_{3}+B_{5}\right) \Pi_{1,1}+\frac{10}{3}\left(B_{1} B_{3}+\frac{1}{2} B_{2}^{2}+B_{4}\right) \Pi_{2,1}+ \\
& +\frac{11}{2}\left(B_{1} B_{2}+B_{3}\right) \Pi_{3,1}+8\left(\frac{1}{2} B_{1}^{2}+B_{2}\right) \Pi_{4,1}+\frac{65}{6} B_{1} \Pi_{5,1}+7 \Pi_{6,1}, \\
& \Pi_{8,4}=\left(\frac{19}{8} B_{1}^{2} B_{2}+\frac{59}{12} B_{1} B_{3}+\frac{29}{12} B_{2}^{2}+\frac{31}{12} B_{4}\right) \Pi_{1,1}+\left(2 B_{1}^{3}+\frac{73}{6} B_{1} B_{2}+\frac{25}{4} B_{3}\right) \Pi_{2,1}+ \\
& +\left(\frac{89}{8} B_{1}^{2}+\frac{45}{4} B_{2}\right) \Pi_{3,1}+\frac{107}{6} B_{1} \Pi_{4,1}+\frac{35}{4} \Pi_{5,1}, \\
& \Pi_{8,5}=\left(\frac{21}{8} B_{1}^{3}+\frac{33}{4} B_{1} B_{2}+3 B_{3}\right) \Pi_{1,1}+\left(\frac{139}{12} B_{1}^{2}+8 B_{2}\right) \Pi_{2,1}+\frac{319}{20} B_{1} \Pi_{3,1}+7 \Pi_{4,1}, \\
& \Pi_{8,6}=\left(\frac{413}{90} B_{1}^{2}+\frac{5}{2} B_{2}\right) \Pi_{1,1}+\frac{153}{20} B_{1} \Pi_{2,1}+\frac{7}{2} \Pi_{3,1}, \\
& \Pi_{8,7}=\frac{223}{140} B_{1} \Pi_{1,1}+\Pi_{2,1} .
\end{aligned}
$$




\section{References}

[1] FCC Collaboration, Eur. Phys. J. C 79, 474 (2019); Eur. Phys. J. ST 228, 261 (2019); 228, 755 (2019); 228, 1109 (2019); A. Blondel et al., arXiv:1906.02693 [hep-ph].

[2] CEPC Study Group, arXiv:1809.00285 [physics.acc-ph]; arXiv:1811.10545 [hep-ex].

[3] ILC Collaboration, arXiv:1306.6327 [physics.acc-ph]; arXiv:1306.6328 [physics.accph]; arXiv:1306.6329 [physics.ins-det]; arXiv:1306.6352 [hep-ph]; arXiv:1306.6353 [physics.acc-ph].

[4] CLICdp and CLIC Collaborations, CERN Yellow Rep. Monogr. Vol. 2 (2018); Vol. 3 (2018); Vol. 4 (2018); Vol. 1 (2019).

[5] Muon g-2 Collaboration, arXiv:1501.06858 [physics.ins-det]; SciPost Phys. Proc. 1, 033 (2019).

[6] E34 Collaboration, JPS Conf. Proc. 8, 025008 (2015); Y. Sato, PoS (KMI2017), 006 (2017); M. Abe et al., PTEP 5, $053 \mathrm{C02}$ (2019).

[7] C.M. Carloni Calame, M. Passera, L. Trentadue, and G. Venanzoni, Phys. Lett. B 746, 325 (2015); G. Abbiendi et al., Eur. Phys. J. C 77, 139 (2017); P. Mastrolia, M. Passera, A. Primo, and U. Schubert, JHEP 11, 198 (2017); S. Di Vita, S. Laporta, P. Mastrolia, A. Primo, and U. Schubert, ibid. 09, 016 (2018); M. Fael, ibid. 02, 027 (2019); M. Alacevich et al., ibid. 02, 155 (2019); G. Venanzoni, PoS (ICHEP2018), 519 (2019); U. Marconi, EPJ Web Conf. 212, 01003 (2019); G. Ballerini et al., Nucl. Instrum. Meth. A 936, 636 (2019); M. Fael and M. Passera, Phys. Rev. Lett. 122, 192001 (2019); P. Banerjee et al., Eur. Phys. J. C 80, 591 (2020); A. Masiero, P. Paradisi, and M. Passera, Phys. Rev. D 102, 075013 (2020).

[8] R. Barate et al. [ALEPH Collaboration], Z. Phys. C 76, 15 (1997); Eur. Phys. J. C 4, 409 (1998); S. Schael et al. [ALEPH Collaboration], Phys. Rept. 421, 191 (2005); M. Davier, A. Hocker, and Z. Zhang, Rev. Mod. Phys. 78, 1043 (2006); M. Davier, S. Descotes-Genon, A. Hocker, B. Malaescu, and Z. Zhang, Eur. Phys. J. C 56, 305 (2008); M. Davier, A. Hocker, B. Malaescu, C. Yuan, and Z. Zhang, Eur. Phys. J. C 74, 2803 (2014).

[9] K. Ackerstaff et al. [OPAL Collaboration], Eur. Phys. J. C 7, 571 (1999); D. Boito et al., Phys. Rev. D 84, 113006 (2011); 85, 093015 (2012); 91, 034003 (2015).

[10] Y. Amhis et al. [HFLAV Collaboration], Eur. Phys. J. C 77, 895 (2017); arXiv:1909.12524 [hep-ex].

[11] J. Bai et al. [BES Collaboration], Phys. Rev. Lett. 84, 594 (2000); 88, 101802 (2002); M. Ablikim et al. [BES Collaboration], Phys. Lett. B 641, 145 (2006); 660, 315 (2008); 677, 239 (2009); Chin. Phys. C 44, 040001 (2020). 
[12] V. Anashin et al. [KEDR Collaboration], Phys. Lett. B 753, 533 (2016); 770, 174 (2017); 788, 42 (2019).

[13] A. Blondel et al., CERN Yellow Reports: Monographs, CERN-2020-003 (CERN, Geneva, 2020); arXiv:1905.05078 [hep-ph].

[14] T. Aoyama et al., Phys. Rept. 887, 1 (2020).

[15] S.L. Adler, Phys. Rev. D 10, 3714 (1974).

[16] A.V. Radyushkin, report JINR E2-82-159 (1982); JINR Rapid Commun. 78, 96 (1996); arXiv:hep-ph/9907228.

[17] N.V. Krasnikov and A.A. Pivovarov, Phys. Lett. B 116, 168 (1982).

[18] A.V. Nesterenko, Strong interactions in spacelike and timelike domains: Dispersive approach, Elsevier, Amsterdam, 222 p. (2017).

[19] T. Appelquist and H. Georgi, Phys. Rev. D 8, 4000 (1973); A. Zee, ibid. 8, 4038 (1973).

[20] R.G. Moorhouse, M.R. Pennington, and G.G. Ross, Nucl. Phys. B 124, 285 (1977); M.R. Pennington and G.G. Ross, Phys. Lett. B 102, 167 (1981); M.R. Pennington, R.G. Roberts, and G.G. Ross, Nucl. Phys. B 242, 69 (1984).

[21] A.A. Pivovarov, Nuovo Cim. A 105, 813 (1992).

[22] A.V. Nesterenko, J. Phys. G 42, 085004 (2015).

[23] A.V. Nesterenko, J. Phys. G 46, 115006 (2019).

[24] P.A. Baikov, K.G. Chetyrkin, and J.H. Kuhn, Phys. Rev. Lett. 101, 012002 (2008); 104, 132004 (2010); P.A. Baikov, K.G. Chetyrkin, J.H. Kuhn, and J. Rittinger, Phys. Lett. B 714, 62 (2012).

[25] P.A. Baikov, K.G. Chetyrkin, and J.H. Kuhn, Nucl. Phys. B (Proc. Suppl.) 189, 49 (2009); P.A. Baikov, K.G. Chetyrkin, J.H. Kuhn, and J. Rittinger, JHEP 07, 017 (2012).

[26] P.A. Baikov, K.G. Chetyrkin, and J.H. Kuhn, Phys. Rev. Lett. 118, 082002 (2017); F. Herzog, B. Ruijl, T. Ueda, J.A.M. Vermaseren, and A. Vogt, JHEP 02, 090 (2017); T. Luthe, A. Maier, P. Marquard, and Y. Schroder, ibid. 10, 166 (2017).

[27] A.V. Nesterenko, Eur. Phys. J. C 77, 844 (2017).

[28] J.D. Bjorken, report SLAC-PUB-5103 (1989).

[29] G.M. Prosperi, M. Raciti, and C. Simolo, Prog. Part. Nucl. Phys. 58, 387 (2007).

[30] K.G. Chetyrkin, A.L. Kataev, and F.V. Tkachov, Phys. Lett. B 85, 277 (1979); M. Dine and J.R. Sapirstein, Phys. Rev. Lett. 43, 668 (1979); W. Celmaster and R.J. Gonsalves, ibid. 44, 560 (1980). 
[31] D.J. Gross and F. Wilczek, Phys. Rev. Lett. 30, 1343 (1973); H.D. Politzer, ibid. 30, 1346 (1973).

[32] W.E. Caswell, Phys. Rev. Lett. 33, 244 (1974); D.R.T. Jones, Nucl. Phys. B 75, 531 (1974); E. Egorian and O.V. Tarasov, Theor. Math. Phys. 41, 863 (1979). 